\title{
CHANGING EPIDEMIOLOGY OF SYSTEMIC FUNGAL INFECTIONS AND THE POSSIBILITIES OF LABORATORY DIAGNOSTICS
}

\author{
ELISABETH NAGY
}

Department of Clinical Microbiology, Albert Szent-Györgyi Medical University, Szeged, Hungary

Advances in medical and surgical therapy over the past two decades have changed the nature of patient care during hospitalization. Recently developed technologies and therapies, involving bone marrow or solid-organ transplants and chemotherapeutic agents, have become common at many medical centers, resulting in the emergence of many immunocompromised individuals. In intensive care units (ICU) the use of invasive monitoring devices, parenteral nutrition, broad-spectrum antimicrobial agents, and assisted ventilation has helped in the treatment of patients suffering from previously devastating or fatal diseases and has provided opportunities for life to premature neonates previously thought to be non-viable [1]. However, these successes have resulted in the proliferation of a severely ill, immunocompromised, long-lasting hospitalized patient population. The AIDS epidemic has also added patients at risk to this growing population of immunocompromised individuals [2]. The immunocompromised patient is highly susceptible to nosocomial infections caused by organisms such as fungi that were previously considered to be of low virulence or "non-pathogenic" [3]. Besides the wellknown endemic fungal pathogens (Histoplasma capsulatum, Coccidioides immitis and Blastomyces dermatitidis), opportunistic Candida species have been implicated most frequently in nosocomial fungal infections.

In the mid-1980s, many institutions, including cancer research, university and community hospitals, reported that fungi were becoming common pathogens in nosocomial infections [3, 4]. In addition, during the period 1980 to 1990 , hospitals in the US providing data to the Center for Disease Control and Prevention (CDC) National Nosocomial Infections Surveillance (NNIS) system reported a steady increase in the rate of nosocomial fungal infections, from 2.0 to 3.8 per 1000 discharges [5]. The high rate of fungal infections was not limited to oncology wards and high-risk nurseries, but also occurred in cardiac surgery and burn and trauma wards. The data suggest that, although nosocomial fungal infections may be more common in large university-affiliated hospitals, the increase in incidence of these infections may be evident in all types of hospitals.

\footnotetext{
ELISABETH NAGY

Department of Clinical Microbiology, Albert Szent-Györgyi Medical University P.O. Box 482, H-6701 Szeged, Hungary
} 
The majority of nosocomial fungal infections are reported to be caused by Candida spp. Even today, $C$. albicans is by far the most common Candida species causing infections in humans, though the prevalence of other Candida spp, such as C. tropicalis, C. parapsilosis, C. krusei, C. lusitaniae and Torulopsis (Candida) glabrata, has increased during recent years. Many institutions have reported newly recognized pathogenic fungi, previously thought to be non-pathogenic, including Malasezzia spp., Fusarium spp., Trichosporon spp., Mucor spp. and Acremonium spp. [6-8]. Aspergillus spp. may also be involved in infections of hematology and oncology patients [4].

C. albicans and the Candida non-albicans species may cause a similar spectrum of diseases, ranging from thrush to invasive diseases such as arthritis, osteomyelitis, endocarditis, endophthalmitis, meningitis or fungemia [1,3].

Candidemia is the most extensively studied nosocomial invasive fungal infection. The presentation of fungemia may vary, with pyelonephritis, peritonitis, hepatosplenic abscesses, pneumonitis, myositis, macronodular skin lesions, osteomyelitis, endophthalmitis, meningitis and/or multiorgan involvement. Most serious nosocomial Candida infections are believed to be endogenous, acquired through prior colonization of the mouth, gastrointestinal tract, vagina or skin, which act as source [9]. The organs involved in disseminated candidiosis may vary with the route of infection. If a previously colonized gastrointestinal tract is the source of infection through a breakdown in mucosal or epithelial tissue, liver and splenic abscesses will probably result [10]. In contrast, if a colonized central venous catheter is the source, endocarditis or renal involvement is more likely [1]. Patients with candidemia and/or disseminated infection usually develop fever and leukocytosis, unless they are also on immunosuppressive medication. It has been proven that some infections caused by non-albicans Candida species such as $C$. lusitaniae or C. parapsilosis or by Aspergillus spp may be exogenous.

The differentiation between the exogenous and the endogenous acquisition of Candida infections is important for determining appropriate control measures to prevent the nosocomial transmission of Candida spp. Strain-typing studies are needed to avoid implicating an environmental source solely on the basis of the presence of a single species of Candida. Interstrain differentiation should be carried out. Strain-typing may also be important in less serious local infections, such as recurrent vulvovaginitis, to distinguish between recurrent or persistent infection. Various typing methods have been developed for this purpose $[11,12]$.

Prevention methods aimed at reducing identified risk factors for nosocomial fungal infection are being increasingly advocated. The usefulness of the prophylactic use of fluconazole among selected leukemia or bone marrow transplant patients is supported by recent studies. For the treatment of invasive Candida and Aspergillus infections, amphotericin B, its liposome-encapsulated form, flucytosine and fluconazole are more widely used. Amphotericin B is the most active and the most toxic antimycotic agent. However, about $80 \%$ of the $C$. lusitaniae strains display resistance to this agent. Flucytosine should be used in combination to prevent the early development of resistance. Fluconazole has a similar activity to that of amphotericin B in invasive fungal infections, with very favourable pharmacokinetics and low toxicity, but C. glabrata and C. krusei are resistant to fluconazole. 
Laboratory methods available for diagnostics of fungal infections

A definitive laboratory diagnosis of invasive fungal infections can be made by the histopathologic demonstration of fungus invaded tissue, or by the isolation of Candida or other fungi from normally sterile body sites. Isolated Candida spp. should be identified at a species level, as some of the non-albicans Candida spp. proved to be resistant to antimycotic agents used in the empirical therapy of invasive fungal infection. Direct microscopic examination of the specimen (liquor) may help to identify encapsulated Cryptococcus neoformans.

For the primary culturing of fungi, Sabouraud dextrose agar is used most frequently. To ensure maximum recovery of fastidious fungi, brain heart infusion (BHI) agar supplemented with $5 \%$ sheep blood and media containing antibacterial agents can also be used with any specimen that might contain normal bacterial flora.

The identification of $C$. albicans can be based on germ tube formation in human serum, and the formation of chlamydospores on the rice agar. C. albicans screen plate (BioMerieux, Sanofi Diagnostics Pasteur) provide a rapid identification of C. albicans isolates directly in clinical material. Non-albicans species can be identified by the classical assimilation/fermentation method, which requires up to 28 days before the identification can be made [13]. For clinical purposes, more rapid tests are needed, which permit species determination on the same day or within 48-72 hours. Specific exoenzymes of the most frequent human pathogenic Candida species can be detected by using CHROMagar (Beckton Dickinson) for the direct culturing of specimens or for the subculturing of non-albicans isolates. The chromogenic substrates incorporated in the agar plate provide well-distinguishable coloured colonies of $C$. albicans (green), C. tropicalis (metallic blue), T. glabrata (purple) and C. krusei (pink). All other species which appear with white colonies can be identified by commercially available identification systems such as the ATB 32C, API 20 CAUX (BioMerieux) strip and the AUXACOLOR, and FUNGSCREEN (Sanofi Diagnostics Pasteur).

To diagnose invasive Candida infections, blood cultures may be used. Several blood culture systems are available for recovery of fungi. The lysis-centrifugation method provides a sensitive method, but several fully automated blood culture systems are also currently available (BacT/Alert, Organon Technica; Bactec, Becton Dickinson; Vital, BioMerieux). Positivity may be recorded after a few hours of incubation. Despite the fact that blood cultures remain the basic tool for the diagnosis of fungemia, this method may fail to detect as many as $50 \%$ of disseminated candidiasis cases. The detection of massive colonization with the same Candida species at different body sites (feces, urine, and oral cavity) may help in the prediction of the dissemination of the Candida infection. Even when blood cultures are positive, identification can be attempted several days after isolation. Serological diagnosis of fungemia is problematic, since it is neither sensitive nor specific. Direct antigen detection from serum samples may also be used for diagnosis of systemic fungal infections. Latex agglutination for Candida, Aspergillus and Cryptococcus (Sanofi Diangnostics Pasteur). However, the sensitivity of these tests is also low. ELISA method for detection of Aspergillus antigen from serum samples and from bronchoalveolar lavage was developed recently and have a sensitivity of $1 \mathrm{ng} / \mathrm{ml}$ (Platelis Aspergillus, Sanofi Diagnostics Pasteur). A PCR-based method can likewise be used to detect pathogenic fungi in human blood [14]. 
Over the past decade, routine susceptibility testing has played a minimal role in the management of nosocomial Candida infections. Many difficulties have arisen as concerning the establishment of a reliable and reproducible susceptibility testing method for Candida spp. Factors such as inoculum size, medium type, and temperature and time of incubation influence the results of such testing, and standardized methods have only recently been developed [15]. A comparison of the relative susceptibilities of Candida spp to antifungal agents, performed in a blinded fashion, revealed great intra- and interlaboratory variations in the results [16]. However, recent studies demonstrated a very good correlation between susceptibility tests involving Etest strips. The National Committee for Clinical Laboratory Standards (NCCLS) [17] provide new guidelines for the susceptibility testing of antifungal drugs for Candida spp.

The increasing number of severe infections caused by different yeasts and other fungi is a great challenge not only for the clinician, but also for the clinical microbiology laboratory. The provision of rapid and reliable methods for these pathogens that are difficult to culture and identify is very important, as at present the clinician's decision must be based on a synthesis of both laboratory and clinical data for the successful management of serious fungal infections.

\section{REFERENCES}

1. Fridkin,S.K., Jarvis,W.R.: Epidemiology of nosocomial fungal infection. Clinical Microbiology Reviews 4 , 499 (1996).

2. Fisher-Hoch,S.P., Hutwagner,L.: Opportunistic candidiasis: an epidemic of the 1980s. Clin Infect Dis 21, 897 (1980).

3. Bodey,G.P.: The emergence of fungi as major hospital pathogens. J Hosp Infect 11(Suppl.A), 411 (1988).

4. Harvey,R.L., Myers,J.P.: Nosocomial fungemia in a large community teaching hospital. Arch Intern Med 147, 2117 (1987).

5. Beck-Sague,C.M., Jarvis,W.R., The National Nosocomial Infections Surveillance System: Secular trends in the epidemiology of nosocomial fungal infections in the United States. J Infect Dis 167, 1247 (1993).

6. Paparello,S.F., Parry,R.I., MacGillivray,D.C., Brock,N., Mayers,D.I.: Hospital-acquired wound mucormycosis. Clin Infect Dis 14, 350 (1992).

7. Welbel,S.F., Mc.Neil,M.M., Pramanik,A., Silberman,R., Oberle,A.D., Midgley,G., Crow,S., Jarvis,W.R.: Nosocomial Malassezia pachydermatis bloodstream infections in a neonatal intensive care unit. Pediat Infect Dis J 13, 104 (1994).

8. Stuart,S.M., Lane,A.T.: Candida and Malassezia as nursery pathogens. Dermatol 11, 19 (1992).

9. Richet,H.M., Andremont,A., Tancrede,C., Pico,J.L., Jarvis,W.R.: Risk factors for candidaemia in patients with acute lymphocytic leucemia. Rew Infect Dis 13, 211 (1991).

10. Cole,G.T., Halawa,A.A., Anaissie,E.J.: The role of the gastrointestinal tract in hematogenous candidiases: from the laboratory to the bedside. Clin Infect Dis 22, S73 (1996).

11. Pfaller,M., Wenzel,R.: Impact of changing epidemiology of fungal infections in the 1990s. J Clin Microbiol Infect Dis 11, 287 (1992).

12. Pfaller,M.A.: Epidemiological typing methods for mycoses. Clin Infect Dis 14(Suppl), S4 (1992).

13. Wickerham,L.J., Burton,K.A.: Carbon assimilation tests for the classification of yeasts. J Bacteriol 56, 363 (1948). 
14. Polanco,A.M., Tudela-Rodríguer,J.L., Suárez-Martínez,J.V. et al.: Detection of pathogenic fungi in human blood by the polymerase chain reaction. EJCMID 14, 618 (1995).

15. Rex,J.H., Pfaller,M.A., Rinaldi,M.G., Polak,A., Galgiani,J.N.: Antifungal susceptibility testing. Clin Microbiol Rev 6, 367 (1993).

16. Galgiani,J.N., Reiser,J., Brass,C., Espinel-Ingroff,A., Gordon,M.A., Kerkering,T.M.: Comparison of relative susceptibilities of Candida spp. To three antifungal agents as determined by unstandardized methods. Antimicrob Agents Chemother 31, 1343 (1987).

17. NCCLS M27-A. Reference Method for Broth Dilution Antifungal Susceptibility Testing of Yeasts; Approved Standard. 1997. 